\title{
Uterine rupture in a primigravida with a term pregnancy: Case report and lessons to learn
}

\author{
R Vatharajh, ${ }^{1}$ MB ChB, FCOG; K Tunkyi, ${ }^{1}$ MB ChB, FCOG; J Devjee, ${ }^{1}$ MBBS, Dip O\&G, MBA; \\ J Moodley, ${ }^{1,2}$ MB ChB, FCOG, FRCOG, MD \\ ${ }^{1}$ Department of Obstetrics and Gynaecology, Addington Hospital, Durban, South Africa \\ ${ }^{2}$ Women's Health and HIV Research Group, Department of Obstetrics and Gynaecology, Nelson Mandela School of Medicine, \\ College of Health Sciences, University of KwaZulu-Natal, Durban, South Africa
}

Corresponding author: J Moodley (jmog@ukzn.ac.za)

Spontaneous uterine rupture (UR) in primigravidas with term pregnancies is a rare occurrence, but is increasing in frequency in high-income countries as a result of a concomitant rise in rates of gynaecological uterine surgery. We present a case from a low- and middle-income country of spontaneous UR at term with no known markers of such an adverse event. The spontaneous UR may have been due to the ingestion of traditional medicines. Health professionals and the community at large must be alerted to the possible dangers of the use of such medications in pregnancy.

S Afr J Obstet Gynaecol 2015;21(1):12-13. DOI: 10.7196/SAJOG.973

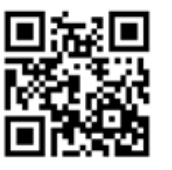

Recently there has been an increasing number of reports from high-income countries of spontaneous uterine rupture (UR) in term primigravidas. These reports relate mainly to women who have had uterine surgery such as hysteroscopic surgery, myomectomies, surgical correction of uterine anomalies and inadvertent uterine perforation. ${ }^{[1,2]}$ We report a case of UR in a primigravida at term, which was probably due to the use of traditional/herbal medications, a common practice in low- and middle-income countries.

\section{Case history}

A 21-year-old primigravida presented to our hospital at 37 weeks' gestation with severe lower abdominal pain and vaginal bleeding. She had clinical and ultrasound features suggestive of hypovolaemic shock and intra-abdominal bleeding, and required an emergency laparotomy.

\section{History of presenting complaint}

On the day of admission to our hospital, the patient reported lack of fetal movements but described what she felt as the baby 'moving up and down' in the upper abdomen. The patient stated that she had ingested half a cup of traditional medication (isiShlambezo; loosely translated this means 'that which cleans') the day before her hospital visit and had noticed increasing intensity of abdominal pain a few hours later. In the last month of her pregnancy she had also ingested a beaten-egg concoction daily, which her family had suggested would help to hasten her labour.

The patient had received antenatal care at our hospital; no abnormalities were detected on physical examination at the first antenatal visit and her basic antenatal laboratory investigations were normal. She had had two non-scheduled visits to our hospital in the last trimester of pregnancy for lower abdominal pains prior to admission. At the first non-scheduled visit she was found to be 36 weeks pregnant by symphysis-fundal height assessment and the fetus was lying in the longitudinal position with the cephalus presenting. At this consultation a diagnosis of false labour was made and the patient was counselled on signs of labour and given an appointment to return in a week's time. On her second non-scheduled hospital visit she also complained of persistent lower abdominal pains and backache. According to early ultrasound findings she was 37 weeks pregnant by gestation and 38 by symphysis-fundal height measurement; pelvic examination revealed the cervical os to be closed. The patient was again thought to be in false labour and asked to return when she was in active labour. At both of these visits the patient had reactive cardiotocographic changes, uterine contractions were not palpable manually, and urine dipsticks were not indicative of a urinary tract infection.

The obstetric history revealed that the pregnancy had been planned and the patient denied any history of previous pregnancies, miscarriages or uterine curettage. She also denied the use of recreational drugs/substance abuse and there was no family history of collagen diseases.

\section{Findings on physical examination at the time of admission}

The patient was pink and alert, with a blood pressure of $96 / 63 \mathrm{mmHg}$ and pulse rate $93 \mathrm{bpm}$, regular and with good volume. The abdomen was distended, and it was difficult to ascertain the symphysis-fundal height. Fetal parts were palpable in transverse lie with free fluid noted in the abdomen. The fetal heart was not audible. The abdomen was peritonitic and blood was noted on the glove following the vaginal examination. Other systems were normal.

\section{Ultrasound findings}

A non-viable extrauterine pregnancy was noted, with free fluid in the abdomen. The uterus measured $97 \mathrm{~mm}$ in its long axis and had an irregular fundal area. The fetus was in the transverse position, and the placenta was in the left side of the abdominal cavity outside the uterine cavity.

\section{Definitive management}

A diagnosis of UR was made, and the patient was resuscitated prior to laparotomy. The findings at laparotomy were a ruptured 
fundus of the uterus not involving the tubes or ovaries; the placenta extruded into the abdominal cavity with intact gestational sac and a macerated stillborn fetus. A haemoperitoneum of approximately $2 \mathrm{~L}$ was noted.

The uterus was repaired in two layers with good haemostasis. The haemoglobin level before surgery was $9 \mathrm{~g} / \mathrm{dL}$ and the patient received 2 units of red cell concentrate following surgery. She made an uneventful recovery and was provided with counselling regarding the stillbirth, and given full information about contraception, future pregnancies and the level of healthcare she needed to seek when pregnant again.

\section{Ethics statement}

Ethical permission was not obtained, as this is a case report. However, verbal informed consent was obtained from the patient after assurance that no identifiers or pictures would be made available. This verbal consent was documented in the hospital records.

\section{Discussion}

UR in primigravidas is rare. ${ }^{[1]}$ Well-known risk factors for UR are a previously scarred uterus following caesarean section, myomectomy and hysteroscopic surgery. ${ }^{[2]}$ Recently, a case report indicated spontaneous UR at 32 weeks' gestation in a patient with an unrecognised Müllerian tract anomaly. ${ }^{[3]}$ Use or abuse of misoprostol for induction of labour has also been reported as a cause of UR in primigravidas. ${ }^{[2]}$

In low- and middle-income countries such as South Africa (SA), it has been found that some patients use traditional medicines containing various decoctions made from medicinal plants to either improve fetal growth or induce labour. In our case, the patient ingested a traditional decoction a day prior to her adverse event and had been eating egg yolk daily for a month. The egg yolk was given to her by her family to facilitate labour. The chemical properties of egg yolk in respect of uterine contractility are unknown and may not have any relation to the UR.

The use of traditional medicines during pregnancy is common in the province of KwaZulu-Natal, SA. Mabina et al. ${ }^{[4]}$ found that $55 \%$ of patients presenting in early labour gave a history of ingesting herbal medications supplied by traditional healers or their families, or bought from medicinal shops or street vendors. Patients who ingested traditional medications (isiShlambezo) had a higher incidence of grade II - III meconium-stained liquor and a higher caesarean section rate than a group that did not ingest herbal concoctions/medications. ${ }^{[4]}$

Although the active chemical agents in herbal medications used during pregnancy in KwaZulu-Natal have still not been identified, it has been postulated that the meconium is due to hyperstimulation and may lead to UR, particularly in parous women. ${ }^{[4]}$ Furthermore, there is evidence that indigenous plants such as Agapanthus africanus and Pentanisia prunelloides, commonly used in traditional herbal remedies during pregnancy and childbirth, may have oxytocic properties..$^{[5]}$

Use of herbal medications in pregnancy in other countries has also been reported. An article on the frequency of UR in Ghana indicates a history of use of herbal medication in patients who had UR in over $50 \%$ of their cases. ${ }^{[6]}$ Given the history of use of traditional medicines by the patient, the lack of risk factors of UR and the fact that she was a primigravida without previous uterine surgery of any type, we postulate that the traditional medicines might have led to uterine hyperstimulation and subsequent UR. Furthermore, there was no evidence of gross anatomical congenital abnormality of the uterus or substance use. There have been case reports of abuse of cocaine and UR. ${ }^{[7]}$ In addition it has been suggested that collagen tissue disorders such as Ehlers-Danlos syndrome are associated with spontaneous UR. ${ }^{[1,2,7]}$

This case illustrates that in low- and middle-income countries in sub-Saharan Africa, healthcare personnel need to be aware of the effects of traditional medicines. In women with lower abdominal pain at term in whom a diagnosis of false labour and abdominal pains not typical of uterine contraction is made, a history of ingestion of traditional medicines must be taken, uterine contractions palpated for, and a cardiotocographic recording done to rule out hyperstimulation.

1. Walsh CA, O'Sullivan RJ, Foley FE. Unexplained prelabour uterine rupture in a term primigravidae. Obstet Gynecol 2006;108(3):725-727.

2. Uccella S, Cromi A, Bogani G, Zaffaroni E, Ghezzi F. Spontaneous prelabour uterine rupture in a primigravidae: A case report and review of the literature. Am J Obstet Gynecol 2011:205(5):e6-8. [http://dx.doi.org/10.1016/j.ajog.2011.08.013]

3. Mizutamari E, Honda T, Ohba T, Katabuchi H. Case report: Spontaneous rupture of an unscarred gravid uterus in a primigravid woman at 32 weeks of gestation. Case Rep Obstet Gynecol 2014(2014), Article ID 209585. [http://dx.doi.org/10.1155/2014/209585]

4. Mabina MH, Pitsoe SB, Moodley J. The effect of traditional medicines on pregnancy outcome. Trop Doct 1997;27(2):84-86.

5. Kaido TL, Veale DJH, Havlik I, Rama DB. Preliminary screening of plants used in South Africa as traditional herbal remedies during pregnancy and labour. J Ethnopharm 1977;55:185-191.

6. Fofie C, Baffoe P. A two year review of uterine rupture in a regional hospital. Ghana Med 2010;44(3):98-102.

7. Gonsoulin W, Borge D, Moise KJ jr. Rupture of the unscarred uterus in primigravid women in association with cocaine abuse. Am J Obstet Gynecol 1990;163(2):526-527. 